\title{
A Theoretical Analysis of Coherent Cross-Peaks in Polarization Selective 2DIR for Detection of Cross- $\alpha$ Fibrils
}

\author{
Dean N. Edun, ${ }^{1, a)}$ Olivia M. Cracchiolo, ${ }^{1, a)}$ and Arnaldo L. Serrano ${ }^{1, b)}$ \\ ${ }^{1}$ Department of Chemistry and Biochemistry, University of Notre Dame, Notre Dame, Indiana 46556, United States
}

\begin{abstract}
The coupled amide-I vibrational modes in peptide systems such as fibrillar aggregates can often provide a wealth of structural information, though the associated spectra can be difficult to interpret. Using exciton scattering calculations, we characterized the polarization selective 2DIR peak patterns for cross- $\alpha$ peptide fibrils, a challenging system given the similarity between the monomeric and fibrillar structures, and interpret the results in light of recently collected 2D data on the cross- $\alpha$ peptide PSM $\alpha 3$. We find that stacking of $\alpha$ helices into fibrils couples the bright modes across helical subunits, generating three new Bloch-like extended excitonic states that we designate $A_{\perp}, E_{\|}$, and $E_{\perp}$. Coherent superpositions of these states in broad-band 2DIR simulations lead to characteristic signals that are sensitive to fibril length, and match the experimental 2DIR spectra.
\end{abstract}

\section{INTRODUCTION}

Infrared (IR) spectroscopy is a useful tool protein secondary structure determination due to its ability to probe backbone amide modes without any added labels. ${ }^{1-3}$ These modes have been thoroughly studied and are well understood for the most common secondary structures. ${ }^{4}$ For example, a mixture of theory and experimental linear vibrational spectroscopy has been used in the past for identifying characteristic features of amyloid fibers, which result from the arrangement of proteins into highly ordered structures. ${ }^{5-8}$ However, little is still known about the IR properties of a recently discovered amyloid structure, which is the subject if this study. Phenol Soluble Modulin $\alpha 3$ (PSM $\alpha 3$ ), a peptide secreted by Staphylococcus aureus, forms amyloid-like structures that appear to play an important role in the structural integrity of bacterial biofilms. PSM 33 adopts a native $\alpha$-helical structure, ${ }^{9} 10$ in its monomeric form (Figure 1a) which it maintains upon fibril formation, making it the first known example of a peptide fibril possessing $\alpha$-helical secondary structure. These fibrils, formed from horizontal stacking (Figure 1b) of $\alpha$-helices about a screw axis of symmetry, have been termed "cross- $\alpha$ fibrils". ${ }^{10,11}$ The formation of these cross$\alpha$ structures raises questions about potential changes in the vibrational spectrum due to increased structural order and potential interhelix coupling. At the same time, the lack of intermolecular hydrogen bonding, a characteristic element of traditional cross-

\footnotetext{
${ }^{a}$ Dean N. Edun and Olivia M. Cracchiolo contributed equally to this work.

b Author to whom correspondence should be addressed. Electronic mail: arnaldo.serrano@nd.edu.
} 
$\beta$ fibrils, raises concerns about the detectability of this species, as it suggests a lack of well aligned, strongly interacting infrared chromophores. The latter is what lends to amide-I FTIR spectra their sensitivity to protein structure.

In this work, we present detailed FTIR and 2DIR spectroscopic calculations in order to determine the dominant modes responsible for the infrared response, as well as to uncover observables in the spectra that would aid in identifying this newly discovered amyloid structure in other systems. We found that, though the small coupling between helices leads to only minor changes in the FTIR spectra, the relative alignment of the IR active modes of the helices and their interactions proved a signature of cross- $\alpha$ fibril formation in polarization sensitive measurements which is sensitive to fibril length and represents coupling between extended helix $A$ and $E$ modes. Finally, we interpret our simulation results in light of recently measured experimental 2DIR data of PSM $33^{12}$

\section{METHODS}

We used a floating oscillator model to calculate the vibrational spectra for the peptides. ${ }^{13}$ Using, coordinates, for both the monomer and fibril, determined from the crystal structures, ${ }^{10}$ we extracted the local transition dipole moments and their corresponding positions in space. ${ }^{13,14}$ We calculated the coupling energies between all pairs of local amide groups using transition dipole coupling (TDC), with parameters from Tasumi and Torii. ${ }^{13,15}$ We used couplings and local site energies to build the one-quantum Hamiltonian. Diagonal entries of the matrix had a center frequency of $1650 \mathrm{~cm}^{-1}$, with additional random gaussian diagonal disorder possessing a standard deviation $12 \mathrm{~cm}^{-1}$, except where otherwise indicated. We diagonalized the one-quantum Hamiltonian to obtain eigenenergies and eigenvectors. ${ }^{15}$ We then calculated exciton transition dipoles by representing them in the local mode basis. FTIR spectra were obtained by scaling each transition by the magnitude squared of its transition dipole moment and summing all modes on a grid and convolving the stick spectrum with a Lorentzian lineshape with a halfwidth of $5 \mathrm{~cm}^{-1}$. A more complete description of the model is included in Appendix A.

Simulations of fibril crystal structures can involve more than 1000 oscillators, causing the vibrational states to be densely packed, making it difficult to choose any particular normal mode as the representative mode for a given frequency region. In order to characterize the main contributions to the spectrum, we used a doorway state representation. To do so, we selected subregions of the spectrum that we wished to characterize. Performing singular value decomposition on a matrix of transition moments of all the eigenmodes within the region provided a unique decomposition of the spectral intensity into 3 orthogonal,

bright doorway modes and n-3 dark modes that represent the set of eigenmodes. ${ }^{16-18}$ Using this approach, we were able to represent peaks in the spectra as originating from select doorway modes. In the present study, we generally analyzed the properties of the brightest of the three doorway modes. A mathematical description of the doorway state analysis is given in Appendix B. 
To simulate the 2DIR spectra, rather than using a more conventional sum over state method, which would require calculation of an intractable number of Feynman diagrams, we followed the exciton scattering approach developed by Mukamel, where the nonlinear response can be modeled by the scattering of two excitons by a local chromophore, under the influence of the local mode anharmonicity, requiring only knowledge of the one-quantum Hamiltonian. ${ }^{19-21}$ Appendix C contains details about the exciton scattering method. While only requiring diagonalization of the one-quantum Hamiltonian, the number of scattering events that must be calculated still scales as $\mathrm{N}^{4}$. The total calculation time required, however, can be significantly shortened by selecting only the pathways which contribute significantly to the spectrum. As shown by Mukamel, exploiting the local nature of the excitation and structural arguments, the actual calculation time can be reduced to $\mathrm{O}\left(\sim \mathrm{N}^{2}\right)$ or even $\mathrm{O}(\sim \mathrm{N}){ }^{19,22}$ To reduce the number of scatterings calculated, we rejected all pairs of excitons with an exciton overlap factor (Eq. C17) below 0.55 . This number was chosen as it is close to the 0.5 cutoff introduced by Mukamel in his demonstration, while still significantly reducing our computation time. ${ }^{22}$ The anharmonicity for all sites was set to $-15 \mathrm{~cm}^{-1}$. Because each simulation included a different number of total oscillators, they required a different number of realizations of diagonal disorder to produce adequate averaging. Table I lists the numbers oscillators in each structure and the number of realizations of diagonal disorder each 2DIR spectrum was averaged over.

TABLE I. Number of realizations of diagonal disorder averaged over for each structure.

\begin{tabular}{ccc|ccc}
\hline \hline $\begin{array}{c}\text { Size of structure } \\
\text { simulated }\end{array}$ & $\begin{array}{c}\text { Number of } \\
\text { oscillators }\end{array}$ & $\begin{array}{c}\text { Number of } \\
\text { realizations }\end{array}$ & $\begin{array}{c}\text { Size of structure } \\
\text { simulated }\end{array}$ & $\begin{array}{c}\text { Number of } \\
\text { oscillators }\end{array}$ & $\begin{array}{c}\text { Number of } \\
\text { realizations }\end{array}$ \\
\hline Monomer & 21 & 20 & 8 -unit cells & 336 & 8 \\
1-unit cell & 42 & 10 & 10-unit cells & 420 & 5 \\
2-unit cells & 84 & 10 & 20-unit cells & 840 & 3 \\
3-unit cells & 126 & 8 & 40-unit cells & 1680 & 2 \\
5-unit cells & 210 & 8 & & & \\
\hline \hline
\end{tabular}

Polarization difference spectra are obtained by normalizing the signals from each of the polarization conditions and subtracting the XXXX spectrum from the XXYY spectrum.

Doorway mode analysis on 2DIR spectra is performed by first simulating 2DIR spectra without diagonal disorder applied. A stricter overlap cutoff of 0.65 was used in these simulations to reduce computation time to a value similar to those with disorder. During the simulation, a list of all combinations of $e_{4} e_{3} e_{2} e_{1}$ involved in calculating the signal (Eq. C8 and C9) was stored, where $e_{n}$ is the exciton resonant with the $n^{\text {th }}$ excitation in the experiment. We filtered for combinations of $e_{1}$ and $e_{2}$, corresponding to specific pathways of interest. By only including pathways where $e_{1}$ and $e_{2}$ have differing fundamental frequencies, we focused on pathways which involved a coherent cross-peak with an oscillation frequency of $\left|\omega_{e_{1}}-\omega_{e_{2}}\right|$ during $T_{2}$. We then stored a list of the transition dipoles involving excitons $e_{1}$ and $e_{2}$ where the oscillation frequency falls within a 
predetermined spectral window. From this list, we perform doorway analysis for both sets of $e_{1}$ and $e_{2}$ excitons, as described above for the linear spectra. The brightest doorway mode from each set were interpreted as excitonic modes that couple, leading to coherent cross peaks oscillating at $\left|\omega_{e_{1}}-\omega_{e_{2}}\right|$. From this, we developed a molecular interpretation of the source of coherent signals in the fibril system.

Simulated waiting time spectra are collected by calculating the 2DIR spectrum for an 8 -unit fibril with $\mathrm{T}_{2}$ values starting from 0 ps incremented by 0.5 to 5 ps. The final set of spectra is averaged over 16 realizations of diagonal disorder. Waiting time coherence oscillation spectra are obtained from Fourier transformation of polarization difference spectra along $T_{w}$. Only the real part of the Fourier transform is plotted.

\section{RESULTS}

\section{A. Simulated spectra of PSMa3 fibers}

To understand how the structure of cross- $\alpha$ fibers influence the spectral properties of the protein, we used previously a solved x-ray structure to simulate infrared spectra. Figure 1a and b show the monomeric and fibril form of the PSM $\alpha 3$ helices, respectively. ${ }^{10}$ The protein fiber has a space group of $\mathrm{P} 2{ }_{1}$ where the proteins within the fiber take on a stacked structure extending along a screw axis. To focus on potential spectral signals associated with the formation of this stacked fiber structure we elected to perform simulations using structures where the coordinates are extended along the screw-axis of the crystal. ${ }^{23}$

Figure 1c shows the simulated FTIR spectra for both the monomeric form of the peptide as well as the fibril form, extended 40 peptide units along the screw-axis. Both spectra were calculated without any diagonal disorder to highlight any changes in the peak shapes. The spectra reveal a small splitting between peaks at around $1650 \mathrm{~cm}^{-1}$ and $1635 \mathrm{~cm}^{-1}$. These peak positions correspond to the frequencies of the $A$ and the doubly degenerate $E_{1}$ normal modes for $\alpha$-helices. For an ideal $\alpha$-helical peptide, these are the only IR active amide-I normal modes. ${ }^{24}$ In the $A$ mode, all peptide groups oscillate in phase and the transition moment is parallel with the axis of the $\alpha$-helix. For short helix lengths, the mode has a slightly blue-shifted frequency compared to the frequency of randomly coiled peptide. ${ }^{16}$ This peak, however, redshifts with increasing helix length and tends to overlap the random coil peak on average. In general, $\alpha$-helix $A$ mode peaks are difficult to distinguish from the amide-I band of randomly coiled peptides using FTIR alone, except in the case of highly hydrated helix backbones. ${ }^{25}$ The weaker doubly degenerate $E_{1}$ modes are made up of amide-I vibrations along the helix with a periodic phase of $2 \pi n / m$ where $n$ is the peptide group index and $m$ is the number of residues per turn. The $E_{1}$ mode has been calculated to have a frequency near $1655,{ }^{26}$ but the peak frequencies can also shift depending on helix length and local disorder. ${ }^{16,27}$ Because the local amide-modes are oriented mostly along the axis of the helix, the $E_{1}$ modes are generally weak. The inability to distinguish important structural information 
about a protein using FTIR alone leads us to examine the structure using 2DIR methods which have proven well suited for examining $\alpha$-helix structures. ${ }^{28-30}$ But first, we analyze the FTIR simulation results using doorway state analysis.

(a)

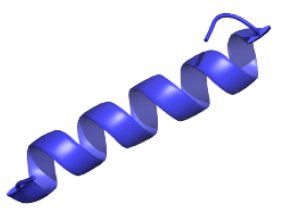

(c)

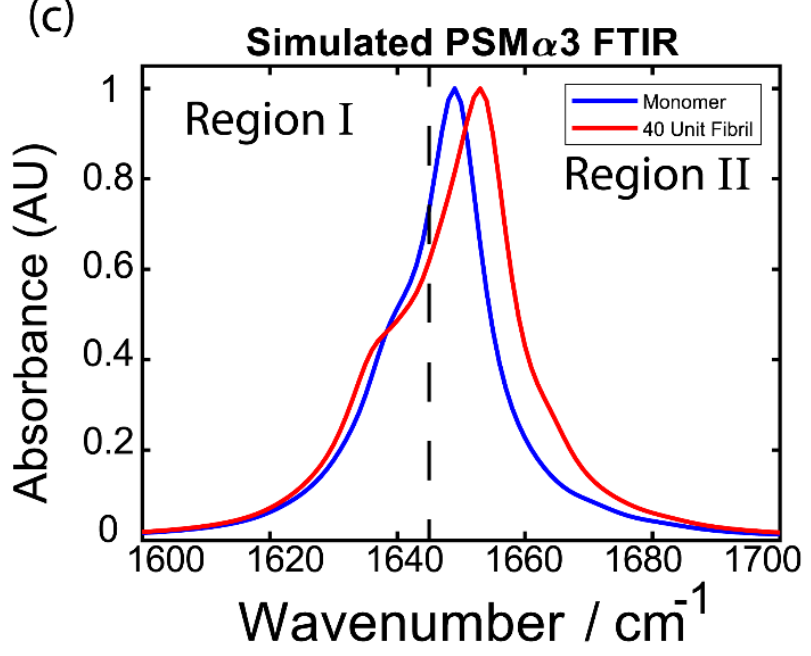

(b)

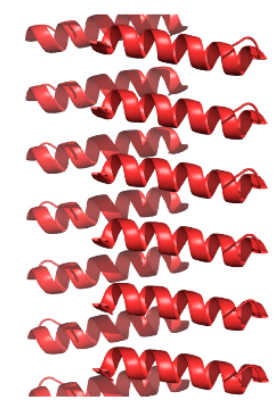




\section{Region I}

Region II

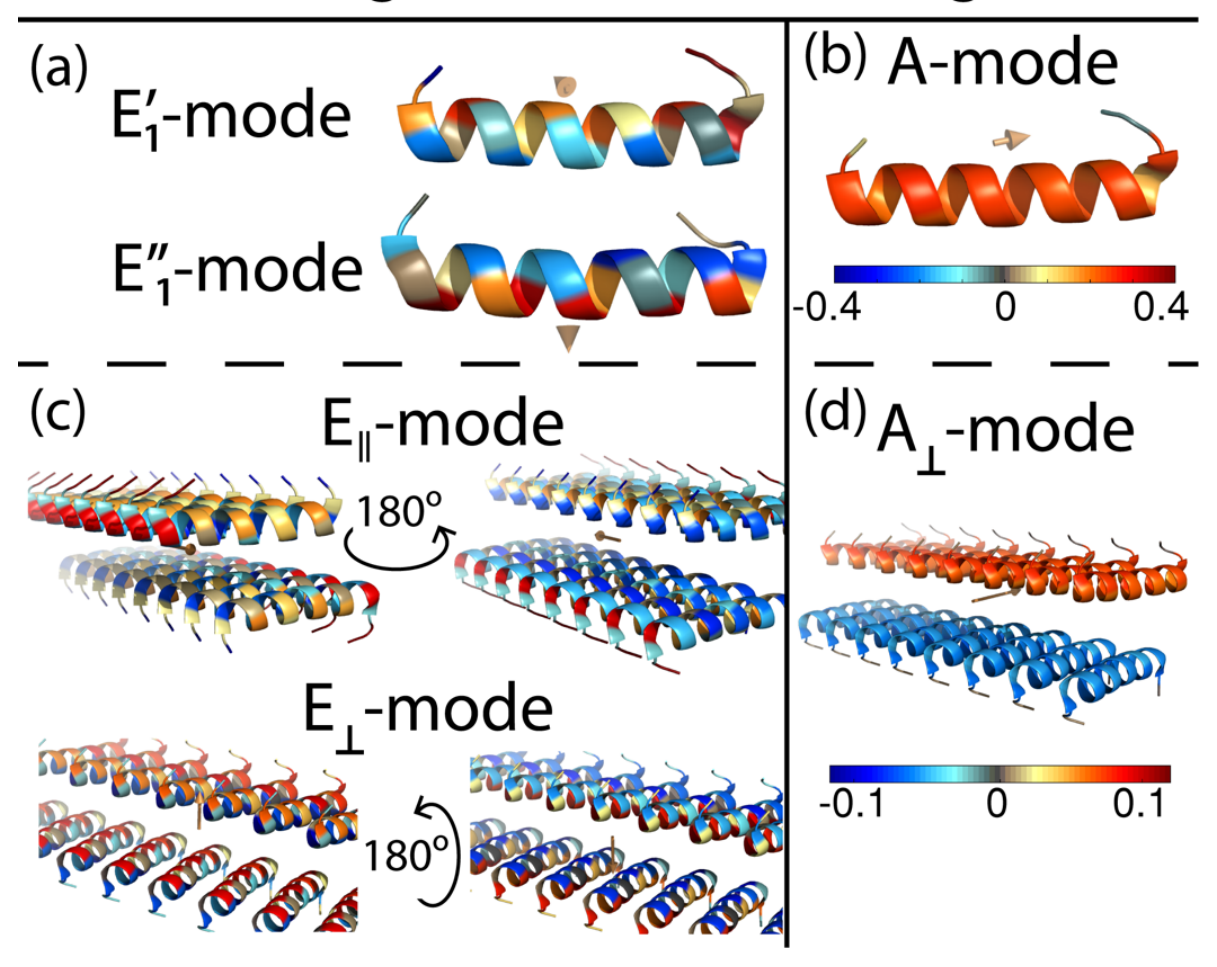

FIG. 2. Doorway mode analysis on the spectra shown in Figure 1. Doorway mode analysis shows the most prominent modes for both the monomer and fiber in the specified regions below and above $1645 \mathrm{~cm}^{-1}$, along with transition dipole vectors (a) and (b) show the E-like and A-like doorway modes for the monomer, respectively. (c) and (d) show the E-like and A-like modes for the fiber, respectively. The color bar shows the amplitude and phase of each residue for each mode.

Applying the doorway analysis on the fibril structure spectrum, the PSM $\alpha 3$ fibril structure's strongest doorway mode above $1645 \mathrm{~cm}^{-1}$, shown in Fig $2 \mathrm{~d}$, is characterized by stacks of $\alpha$-helical $A$-modes, where the two sheets that make up the fibril have opposite phase to one another. As the two sheets of helices have opposite orientations, the total transition dipole moment for both sheets are in the same direction resulting in a strong overall transition dipole moment that is perpendicular the fibril screw axis. This extended $A$-like mode clearly results from coupling between helical subunits. We designate this mode $A_{\perp}$ to indicate that the transition dipole is perpendicular to the fibril axis. Figure $2 \mathrm{c}$ shows that for the region below $1645 \mathrm{~cm}^{-1}$, the 2 brightest states have an $E_{1}$ character, one of which has a transition moment that runs parallel to the fibril axis. We designate this mode $E_{\|}$, to indicate that its transition moment is parallel to the fibril axis. The other has a transition moment that is perpendicular to the other two, which we designate $E_{\perp}$. The expectation values for the energy of these modes, using $A_{\perp}$ as an example, is calculated as $\left\langle A_{\perp}|\widehat{H}| A_{\perp}\right\rangle$, where $\widehat{H}$ is the system Hamiltonian defined in Eq. A5 and $\left|A_{\perp}\right\rangle$ is the doorway mode in the local mode basis. We found this expected energy value to be equal to $1653 \mathrm{~cm}^{-1}$ for $A_{\perp}, 1646 \mathrm{~cm}^{-1}$ for $E_{\perp}$, and $1642 \mathrm{~cm}^{-1}$ for $E_{\|}$. 
We turn to 2DIR calculations to further investigate the inter-helix vibrational coupling, as 2DIR is especially sensitive to coupling. ${ }^{31}$ Although expected shifts in frequencies due to intramolecular interactions between the localized $A$ and $E_{1}$ modes are small, the effects of coupling can be teased out of 2DIR measurements using the polarization sensitivity of cross peaks. It is important to note that the transition dipoles of the extended $A_{\perp}, E_{\|}$, and $E_{\perp}$ modes are very nearly perpendicular to one another, making polarization well-suited for this analysis. We simulated polarization dependent 2DIR measurements to isolate cross-peaks in the spectrum and probe relative orientation between transition moments. This can be used to isolate cross peaks even from nearly degenerate vibrational modes. ${ }^{28,32,33}$

The diagonal peak signals in the $\mathrm{XXXX}$ (i.e., pump-probe parallel polarization) are three times larger than the diagonal peaks in the XXYY (pump-probe perpendicular) signal. The ratio between the cross-peak signals in the XXXX and XXYY polarizations, however, depends on the angle between the modes and the polarization of incident light. The ability to select out different pathways through polarization control is important as it can allow us to remove diagonal contributions from the spectrum. ${ }^{34,35}$ Because the frequency splitting between $A$ and $E_{1}$ modes in $\alpha$-helices is relatively small, peaks along the diagonal obscure cross-peaks. By calculating polarization difference spectra, we can eliminate these obscuring signals.

Simulated Monomer PSMa3 Broad Band Pump 2DIR Spectrum

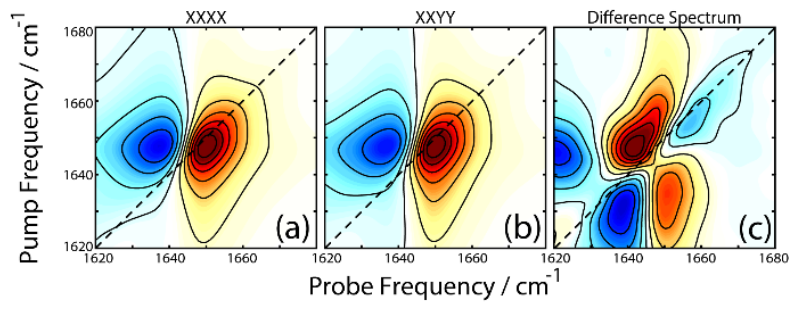

Simulated Monomer PSMa3 Narrow Band Pump 2DIR Spectrum

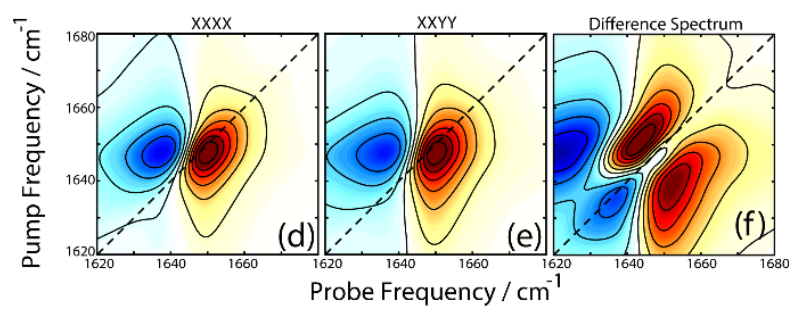

FIG. 3. Simulated 2DIR spectra of monomeric PSM $\alpha 3$ using broad band (top) and narrow band (bottom) pump conditions. The XXXX, $\mathrm{XXYY}$, and difference spectrum was calculated for each. The difference spectra reveal cross-peaks consistent with the $A$ and $E$ mode coupling of $\alpha$-helices.

Figure $3 \mathrm{a}$ and $\mathrm{b}$ shows simulated 2DIR spectra of monomeric PSM $\alpha 3$ for XXXX and XXYY polarizations, respectively. The spectra agree well with the 2DIR spectrum for a purely $\alpha$-helical peptide, such as the spectrum in Figure $1 \mathrm{~d}$ of reference $28 .^{28}$ We normalized both spectra to their peak amplitude then subtracted the parallel signal from the perpendicular. Figure $3 \mathrm{c}$ 
shows the simulated difference spectrum, displaying what appears to be two cross peak pairs at pump frequencies 1635 and $1647 \mathrm{~cm}^{-1}$, close to the frequencies for the $\alpha$-helix $A$ and $E_{1}$ modes, respectively. ${ }^{16}$

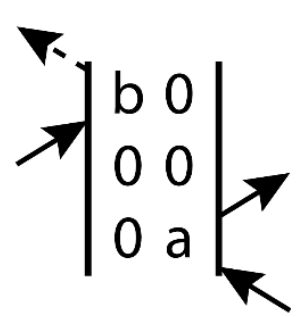

(a)
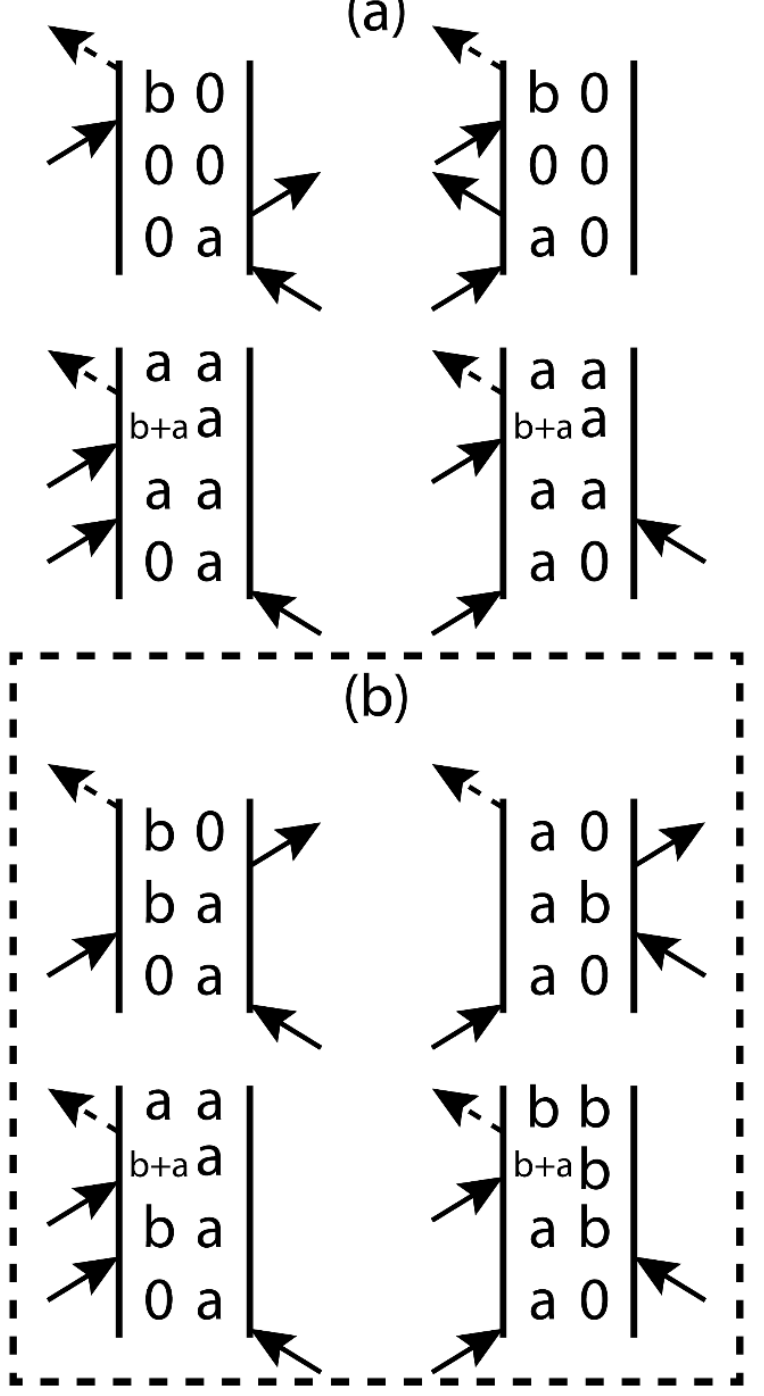

FIG. 4. Double-Sided Feynman diagrams showing all possible cross-peak pathways. The first four pathways (a) are allowed in the narrow band experiment and come in rephasing and non-rephasing pairs, leading to absorptive features in the spectra whereas the four pathways in the box (b) are not allowed in the narrow band experiment, and all lack a phase conjugate partner, leading to non-absorptive features. All eight pathways are allowed in the broad band experiment.

By calculating all Feynman pathways, as featured in Figure 4, though, we have implicitly calculated the broad band pump version of the 2DIR experiment, as we allow for the excitation of two excitons with two different frequencies during the pump interactions. It is sometimes convenient, however, to collect 2D spectra using a narrow band pump where the first two interactions occur at the same (or similar) frequencies. This restricts the set of pathways sampled only to those where there is no coherent state during the waiting time, $T_{2}$, eliminating non-absorptive cross-peak features, some of which lie along the diagonal. This reduces the overall complexity of the 2DIR spectrum while still retaining much of the useful cross-peak information 2DIR provides. ${ }^{36}$ Figures $3 \mathrm{~d}-\mathrm{f}$ show the spectra for the monomer using this narrow band approach. While the 
polarization control simulations remain almost identical to their broad band pump counterparts, cross-peak pair identification is easier using the narrow band approach. Similar peak pairs have been reported for narrow band pump 2DIR measurement of model helical peptides. ${ }^{28,37,38}$

Simulated PSMa3 Fiber Broad Band Pump 2DIR Spectrum

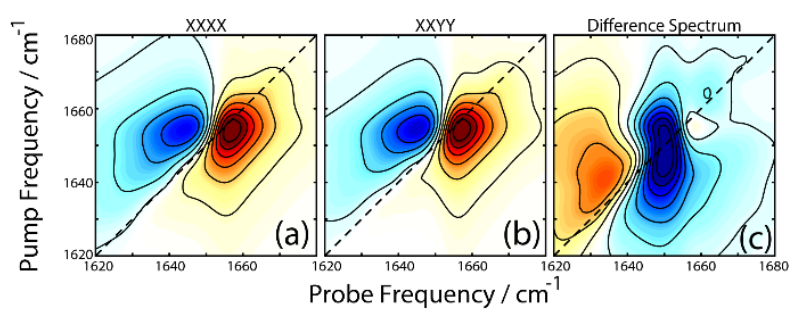

Simulated PSMa3 Fiber Narrow Band Pump 2DIR Spectrum

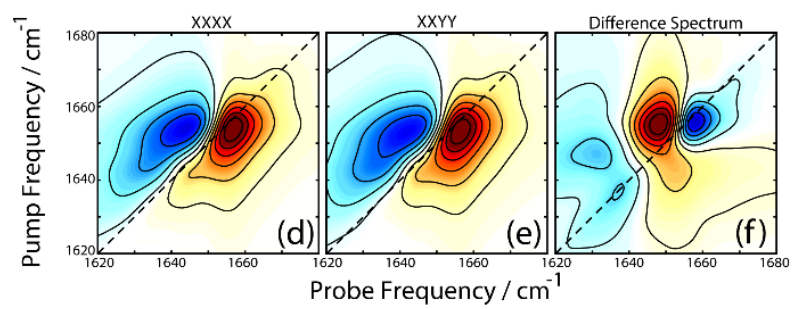

FIG. 5. Simulated 2DIR spectra of PSM $\alpha 3$ fibers extended 20-unit cells (40 peptides) along the screw-axis using broad band (top) and narrow band (bottom) pump conditions. The narrow band difference spectrum has a similar cross-peak pattern to that of the monomer whereas the broad-band spectrum has a new peak pattern along the diagonal.

We also applied this method on a 20-unit cell (40 peptide) PSM $\alpha 3$ fibril structure. The Figures 5a-c show the spectra using the broad band approach. The difference spectrum displays a set of peaks with no clear peak pairs. The change between Figures $3 \mathrm{c}$ and $5 \mathrm{c}$ indicates that fibril formation has some effect on the spectrum, but it is unclear where the change originates from, though overall appearance is that of a non-rephasing signal on the diagonal, which may result from "diagonal" cross peaks pathways of the sort seen in the right column of Figure 4b. Additionally, we also performed simulations using the narrow band approximation shown in Figures 5d-f. This difference spectrum shows the return of the $A$ and $E_{1}$ coupled cross-peaks, though with differing peak ratios compared to the monomer. The difference between the broad band and narrow band difference spectra suggest that new diagonal coherent cross-peaks in the spectra result from coherent coupling between the fibril modes. 


\section{Simulated PSMa3 Broad Band Pump 2DIR Spectra with Increasing Fiber Length}

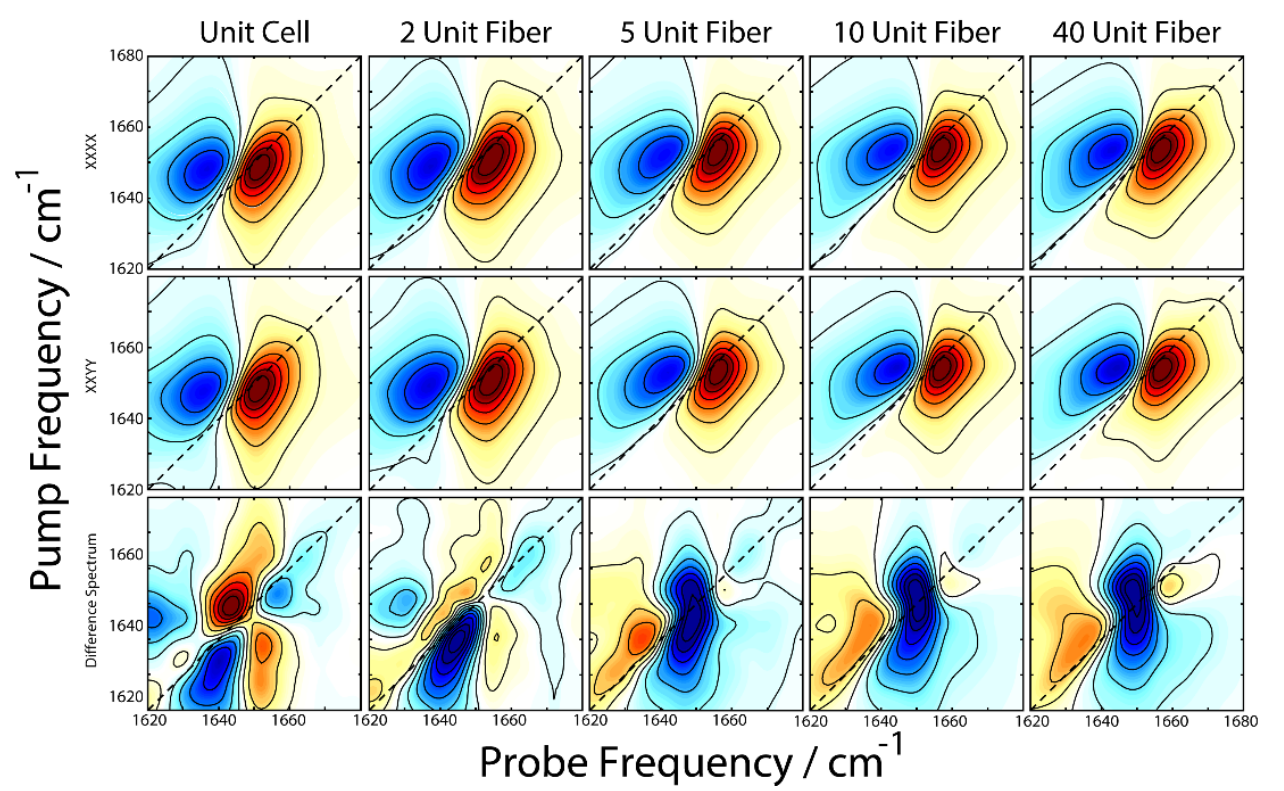

FIG. 6. Simulated PSM $\alpha 3$ calculated using XXXX and XXYY polarizations with increasing fiber lengths. The bottom row displays the difference spectra for all fiber lengths. As more units are added, the center blue vertical shaped peak becomes more prominent.

To test whether the cross-peak pattern in Figure $5 \mathrm{c}$ arose due to through-fibril interactions, we simulated the 2DIR spectra with increasing length of PSM $\alpha 3$ fibril, starting with a single unit cell consisting of two repeats of the peptide, to a 40-unit cell fibril consisting of 80 peptide repeats. The simulation results in Figure 6 show that the main diagonal peaks in the XXXX spectra remain mostly unchanged with increasing fiber length, but the underlying cross-peaks seen in the difference spectra gradually shifts from the pattern seen for the monomer in Figure $3 \mathrm{c}$ to a peak pattern consistent with the non-rephasing 2DIR lineshape pattern of the fibril, which would be expected from coherent cross-peaks arising from interactions along the fibril axis. $^{35}$

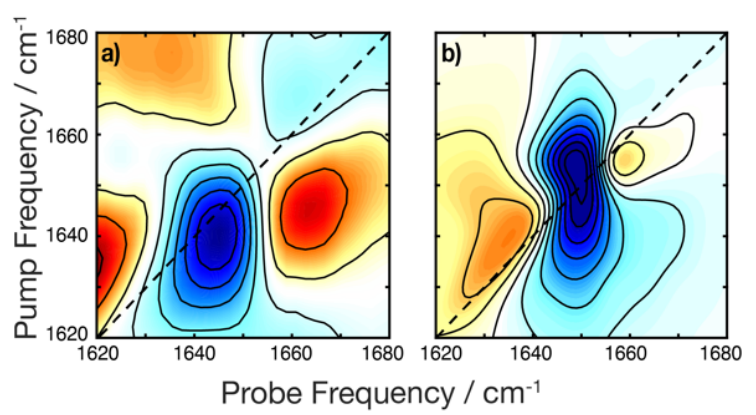

FIG. 7. Comparison of (a) experimental 2DIR difference spectrum of PSM $\alpha 3$ fibers $^{39}$ vs (b) simulated 2 DIR difference spectrum of a 40 unit fiber PSM $\alpha 3$. 
Our previous study examining the experimental 2DIR spectra of PSM $\alpha 3$ fibers produced a polarization difference spectrum (Figure 7a) that agrees with our simulated difference spectrum in Figure $7 \mathrm{~b}$. The long vertical blue peak appears to result from the coupling of modes within the fibril state.

\section{B. Molecular origin of the characteristic cross- $\alpha$ signal}

To explore the molecular origin of the coherent peaks along the diagonal that appear to be characteristic of cross- $\alpha$ structures, we simulated the spectrum of a 5-unit fiber without diagonal disorder, filtering out any peaks which did not contribute to a coherent signal. Specifically, when calculating the rephasing signal, rather than summing over all combinations of $e_{4} e_{3} e_{2} e_{1}$ in Eqs. C8, we included only contributions where $e_{1}=e_{3}, e_{2}=e_{4}$, and $e_{1} \neq e_{2}$. Likewise, when calculating the non-rephasing spectrum, the sum included only contributions where $e_{1}=e_{4}, e_{2}=e_{3}$, and $e_{1} \neq e_{2}$. This filtered coherent-only spectrum allowed us to determine the normal modes responsible for the coherent signal along the diagonal of the crosspolarization difference spectrum. For simplicity, we refer to the mode that undergoes the first interaction, $e_{1}$, as mode $a$ and the mode that undergoes the second interaction, $e_{2}$, as mode $b$. The effect of these filtering conditions is that we only sum over pathways which involve a coherence along $T_{2}$, corresponding to the Feynman diagrams shown in Figure 8 . The range of coherence frequencies, $\left|\omega_{a}-\omega_{b}\right|$, was limited to 2 to $16 \mathrm{~cm}^{-1}$ to ensure that any coherences between the $A_{\perp}, E_{\|}$, and $E_{\perp}$ modes were captured, as the differences in their expected energy values, reported in section III.A above, fall in this range.

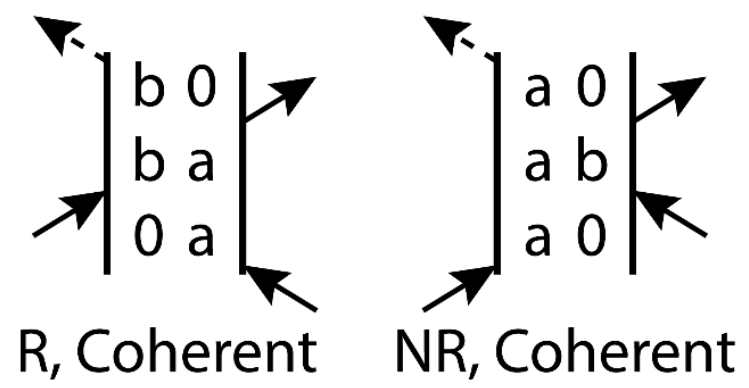

FIG. 8. Feynman diagrams showing both rephasing and non-rephasing coherent pathways. These pathways undergo oscillations of $\mid \omega_{a}-$ $\omega_{b} \mid$ during $T_{2}$. 


\section{Coherent Signals $\left|\omega_{\mathrm{a}}-\omega_{\mathrm{b}}\right|=2$ to $8 \mathrm{~cm}^{-1}$}

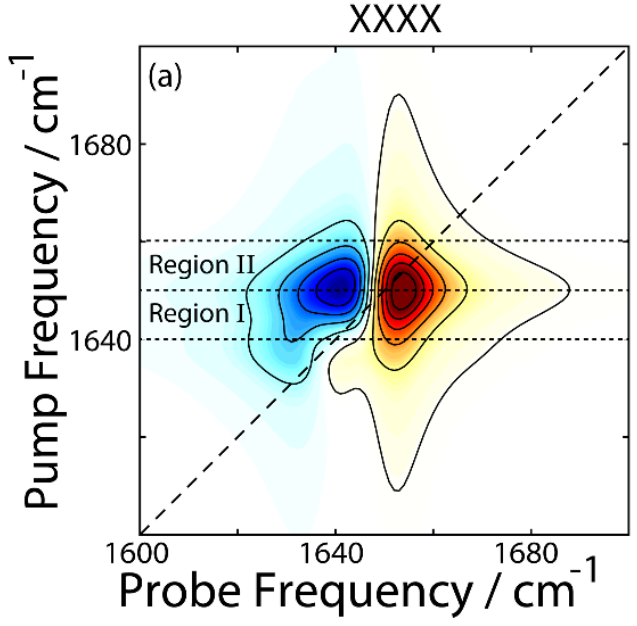

(b)
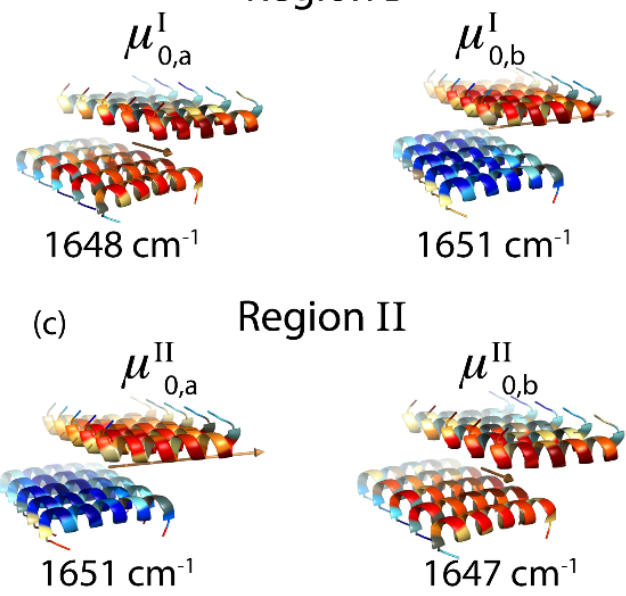

FIG. 9. (a) Filtered, coherence-only spectrum, as described in the main text, calculated from coherent signals where the difference frequency between the two modes is 2 to $8 \mathrm{~cm}^{-1}$. (b) The first doorway mode calculated using the coupled transition dipoles in region I. $\mu_{0, a}^{I}$ is the doorway state from the transition dipoles calculated from the first excited mode whereas $\mu_{0, b}^{I}$ is the transition dipole calculated from the second excited mode. (c) The first doorway modes of all pairs of coupled modes in region II.

In Figure 9a, we plotted the filtered coherence-only spectrum where $2 \leq\left|\omega_{a}-\omega_{b}\right|<8 \mathrm{~cm}^{-1}$. The choice of a cutoff of 8 $\mathrm{cm}^{-1}$ was motivated by the expectation values found above for the extended modes $A_{\perp}\left(1653 \mathrm{~cm}^{-1}\right), E_{\perp}\left(1646 \mathrm{~cm}^{-1}\right)$, and $E_{\|}(1642$ $\mathrm{cm}^{-1}$ ), as we expected coherences between $A_{\perp}$ and $E_{\perp}$ to lie in this region, and coherences between $A_{\perp}$ and $E_{\|}$to lie in the 8-16 $\mathrm{cm}^{-1}$ range, which is plotted in Figure 10. The coupling between modes is obscured in these diagonal cross peaks due to the signal resonating at the same frequency, $\omega_{a}$, during both $\tau_{1}$ and $\tau_{3}$. Typically, one could perform a waiting time study along $T_{2}$ to determine the difference frequency between $\omega_{a}$ and $\omega_{b}$ and thus backtrack to determine the coupled modes. Here, however, we can determine the coupled modes directly by keeping a list of all pairs of modes $a$ and $b$ that contribute to this signal and doing doorway analysis on the two sets of transition moments, $\left\{\mu_{a}\right\}$ and $\left\{\mu_{b}\right\}$. In Figure 9b, we present the brightest $a$ and $b$ doorway modes from the region of the spectrum from 1640 to $1650 \mathrm{~cm}^{-1}$ and map the resulting amplitudes and phases 
of the doorway modes onto the crystal structure. This analysis reveals that the signal in Region I of the filtered coherent spectrum in Figure 9a original form coupling between the $A_{\perp}$ and $E_{\|}$modes. Repeating the doorway analysis on the region from 1650 to $1660 \mathrm{~cm}^{-1}$, shown in Figure 9c, gives similar doorway modes in reverse order.

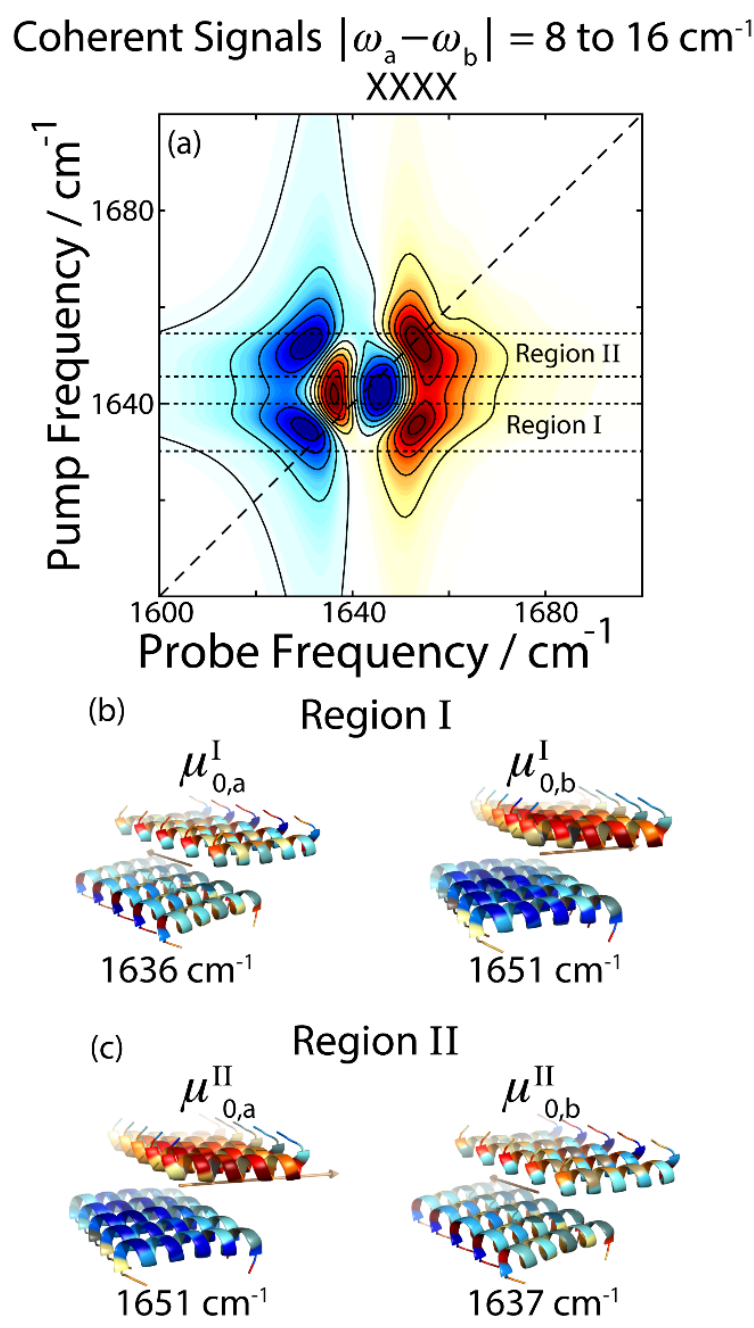

FIG. 10. (a) Filtered, coherence-only spectrum calculated from coherent signals where the difference frequency between the two modes is 8 to $16 \mathrm{~cm}^{-1}$. (b) The first doorway mode calculated using the coupled transition dipoles in region I. $\mu_{0, a}^{I}$ is the doorway state from the transition dipoles calculated from the first excited mode whereas $\mu_{0, b}^{I}$ is the transition dipole calculated from the second excited mode. (c) The first doorway modes of all pairs of coupled modes in region II.

Repeating the calculations for interactions where $8 \leq\left|\omega_{a}-\omega_{b}\right|<16 \mathrm{~cm}^{-1}$ revealed a new peak pattern in Figure 10a. The doorway mode analysis of the first and second excitations as described above were repeated and the results plotted in Figure $10 \mathrm{~b}$ and $\mathrm{c}$, from which it can be seen that the origin of these peaks is also coupling between $A_{\perp}$ and $E_{\|}$modes. No evidence of coherent signal originating from coupling to the $E_{\perp}$ was found. It's possible that such an interaction would be forbidden for symmetry reasons, though this would require further theoretical efforts in the future. The present analysis does 
support the view, though, that interactions between the newly formed delocalized states in the cross- $\alpha$ structure lead to the diagonal signature in Figure 7.

\section{Time dependence of diagonal coherences}

Because the $A_{\perp}$ and $E_{\|}$modes of the fibril appear to be coherently coupled, we should expect the coherent diagonal cross-peaks to oscillate along the $T_{2}$ axis. We performed simulations of the 8-unit fiber with waiting times ranging from 0 to 5 ps in 0.5 ps increments. The difference spectra for select timepoints are plotted in Figure 11a. The diagonal cross-peaks evolve with increasing waiting times which is what we would expect for coherent cross-peaks. During early time points, the difference spectrum is dominated by contributions along the diagonal. The later timepoints, however, are almost completely dominated by static cross-peaks and the difference spectrum effectively appears like the monomer difference spectrum. This rapid loss of coherent signals can be explained by the fact the $A_{\perp}$ and $E_{\|}$modes are each really a superposition of closely spaced but nondegenerate energy eigenstates, the frequencies of which quickly go out of phase with one another, leaving behind only static cross peaks.

(a) Simulated PSMa3 Waiting Time 2DIR Spectra 8 Unit Fiber

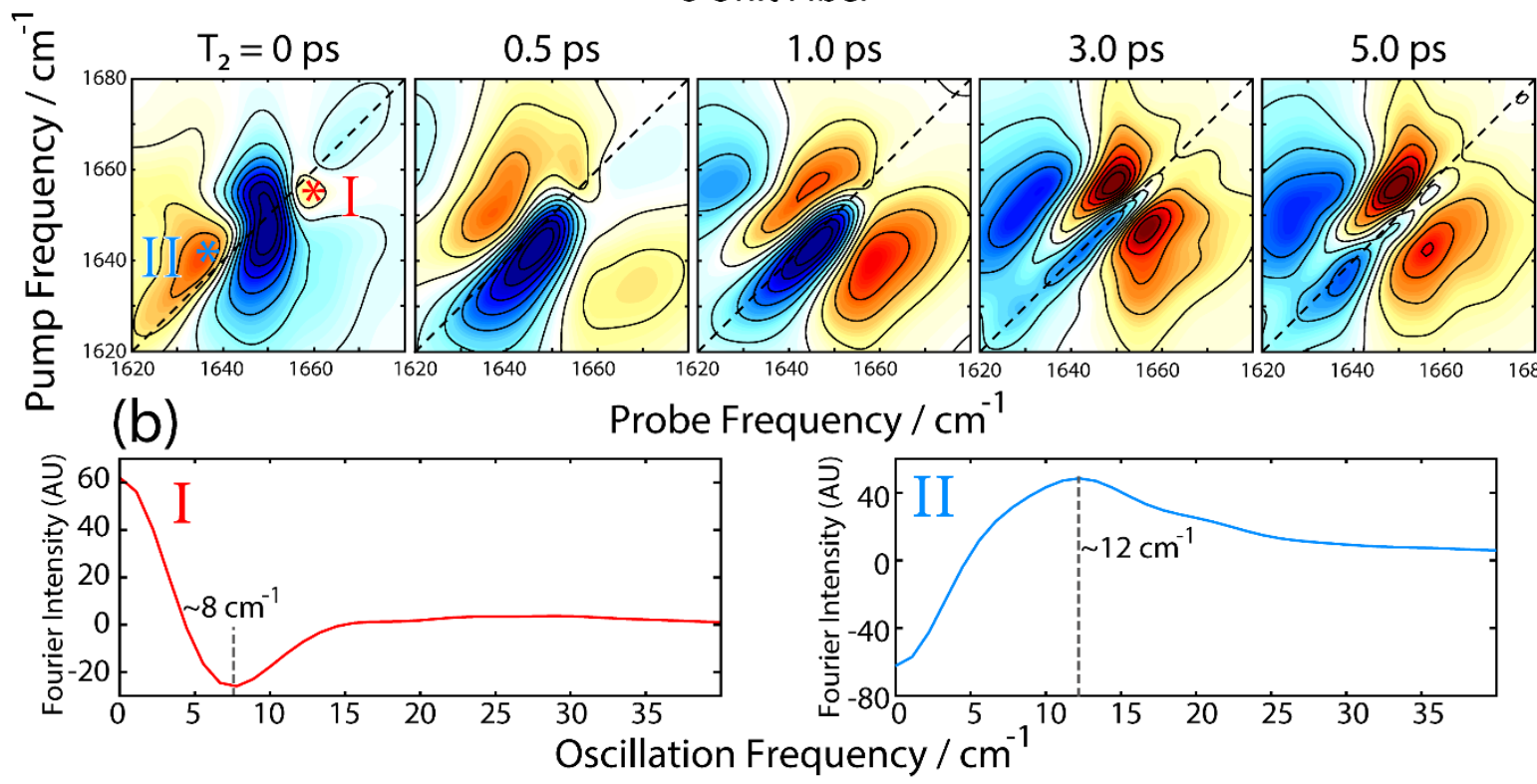

FIG. 11. (a) Simulated difference spectra of an 8-unit fiber calculated at the indicated $\mathrm{T}_{2}$ waiting times. (b) Fourier transforms of two slices of the spectrum, sliced through the indicated pixels along the $\mathrm{T}_{2}$ dimension.

To pick out the coherence frequency along $T_{2}$, we picked two points from the spectra and took a slice of those points along the $T_{2}$ axis. We performed Fourier transformation of the slice to determine the oscillation frequencies present, similar to what has been done to extract coherence frequencies in Two-Dimensional Electronic Spectroscopy (2DES).$^{40}$ Figure $11 \mathrm{~b}$ shows the Fourier transforms for two slices taken at the indicated points. The spectrum for the slice taken from the point I at about 
$1655 \mathrm{~cm}^{-1}$ on the pump axis is weakly peaked at around $8 \mathrm{~cm}^{-1}$, while the spectrum generated from point II is peaked near 12 $\mathrm{cm}^{-1}$. These values roughly agree with the differences between expected energy values for the doorway states $A_{\perp}, E_{\perp}$ and $E_{\|}$.

\section{DISCUSSION}

The sets of simulation methods we performed all point to the emergence of a characteristic coherent cross-peak signal of PSM $\alpha 3$ cross- $\alpha$ fibers, emerging from interactions between newly formed extended modes of the fibrils. Analyzing differences between narrow -band and broad-band spectra can allow one to discriminate between coherent signals in the 2DIR spectrum. Furthermore, we can determine the source of the coherences in simulations directly through filtering out non-coherent signals and examining the modes responsible for the remaining signal. Using waiting time simulations, we can confirm the frequencies of the coherent oscillations in the signal are correlated to the coupled modes we determined previously.

The shape of the coherent cross-peaks appearing in the polarization difference spectra of cross- $\alpha$ fibrils is that of a nonrephasing lineshape. The non-rephasing origin of the peaks causes the three lobed structure seen in Figure $7 \mathrm{~b}$. Generally, coherent cross-peaks arising from rephasing pathways will dominate the off diagonal regions of polarization difference 2DIR spectrum, while non-rephasing coherent cross-peaks dominate the diagonal regions. ${ }^{35}$ The appearance of the strong nonrephasing signal along the diagonal of the spectra in Figure 7 is thus not surprising.

Deep analysis on the origin of coherent peaks has been sparse within the 2DIR community, limited to small molecules and localized sites where there are a handful of total pathways to consider. ${ }^{41,42}$ Analysis of cross-peaks and differences between rephasing and non-rephasing spectra, however, has been closely explored in 2DES, especially in the case of supra-molecular light harvesting systems, where examination of quantum beating and non-rephasing signals have been used to determine mechanism of energy transfer and relaxation in congested spectra. ${ }^{43-45}$ For example, many 2DES studies have leveraged details of the coherences in light harvesting complexes to determine that long lived quantum beats have vibrational origins. ${ }^{46-48}$ In another study involving artificial light harvesters aligned via a wire guide exhibits quantum beating as well as a more intense non-rephasing peak compared to the rephasing peak. ${ }^{49} \mathrm{Here}$, we have employed methods similar in spirit to determine the molecular origin of 2DIR signals of cross- $\alpha$ fibrils. We have shown it is possible to obtain insights into the vibrational coherences in super-molecular structures and that careful examination of these coherent peaks may prove to be useful in resolving super-molecular structure in the future.

\section{CONCLUSION}

In conclusion, we have used variety of IR spectrum simulation techniques to decode the infrared spectra of cross- $\alpha$ fibrils, a structure with many oscillators and a relatively featureless FTIR and absorptive 2DIR spectrum. We simulated both narrowband and broadband polarization selective 2DIR spectra of PSM $\alpha 3$ fibers using the nonlinear exciton scattering 
simulation approach. We identified three new characteristic extended vibrational exciton states characteristic of cross- $\alpha$, the $A_{\perp}, E_{\perp}$, and $E_{\|}$modes. We also showed that a combination of polarization selectivity and narrowband/broadband spectra allowed us to identify and characterize a coherent cross peak between these new modes that is characteristic of cross- $\alpha$ fibrils. We believe that chracterization of this signature may allow future identifications of cross- $\alpha$ structure in solution phase measurements. We also believe that the approach presented, combining polarization selectivity, time and frequency domain measurements, and doorway state analysis, can be a fruitful strategy for investigating molecular origins for congested spectra of other supramolecular complexes.

\section{APPENDIX}

\section{A. One-quantum site-energy model}

The Hamiltonian $\widehat{H}$ for a quantum system can be written as

$$
\widehat{H}=\widehat{H}_{0}+\widehat{H}^{\prime},
$$

where $\widehat{H}_{0}$ is the Hamiltonian of the molecular aggregate and $\widehat{H}^{\prime}$ describes the interactions between the molecules and the light. For a molecular aggregate with energy levels $\varepsilon_{e}$ and eigenstates $\left|\psi_{e}\right\rangle$, the time independent Schrodinger equation is

$$
\widehat{H}_{0}\left|\psi_{e}\right\rangle=\varepsilon_{e}\left|\psi_{e}\right\rangle
$$

The external electric field $\boldsymbol{E}(t)$ of the light pulses in the 2D experiment acts on the system through the dipole operator $\widehat{\boldsymbol{\mu}}$,

$$
\widehat{H}^{\prime}(t)=-\widehat{\boldsymbol{\mu}} \cdot \boldsymbol{E}(t) .
$$

The dipole operator takes a vibrational mode up or down an energy level

$$
\widehat{\boldsymbol{\mu}}=\sum_{m}\left(b_{m}^{\dagger}+b_{m}\right) \boldsymbol{\mu}_{m}
$$

where $b_{m}^{\dagger}$ and $b_{m}$ are the creation and annihilation operators for local mode $m$, respectively, and $\boldsymbol{\mu}_{m}$ is the transition dipole moment of local mode $m$. The full Hamiltonian can be written in the local mode basis as

$$
\widehat{H}(t)=\sum_{m, n} \delta_{m n} \varepsilon_{m} b_{m}^{\dagger} b_{m}+\left(1-\delta_{m n}\right) \beta_{m n} b_{m}^{\dagger} b_{n}-\sum_{m}\left(b_{m}^{\dagger}+b_{m}\right) \boldsymbol{\mu}_{m} \cdot \boldsymbol{E}(t)
$$

where $\varepsilon_{m}$ is the energy of local mode $m$ and $\beta_{m n}$ is the coupling energy between local modes $m$ and $n$.

To obtain the local mode frequencies, we modeled each amide bond in the structure as a floating oscillator and assigned it a frequency of $1650 \mathrm{~cm}^{-1}$ plus a random offset, to produce gaussian diagonal disorder, to model the frequency of a free amide- 
I mode. We made use of a transition dipole coupling (TDC) model to calculate the coupling energies between each local mode. Here, the coupling energies are calculated as

$$
\beta_{m n}=\frac{|\mu|^{2}}{4 \pi \epsilon_{0}}\left[\frac{\hat{\mu}_{m} \cdot \widehat{\mu}_{n}-3\left(\hat{r}_{m n} \cdot \widehat{\mu}_{m}\right)\left(\hat{r}_{m n} \cdot \widehat{\mu}_{n}\right)}{r_{m n}^{3}}\right],
$$

where $|\mu|$ is the strength of an amide-I transition dipole moment, $\epsilon_{0}$ is the vacuum permittivity, $\widehat{\boldsymbol{\mu}}_{m}$ is the unit vector describing the orientation of transition dipole $m, \hat{\boldsymbol{r}}_{\boldsymbol{m} \boldsymbol{n}}$ is the unit vector of the separation between $\boldsymbol{\mu}_{m}$ and $\boldsymbol{\mu}_{n}$, and $r_{m n}$ is the distance between $\boldsymbol{\mu}_{m}$ and $\boldsymbol{\mu}_{n}$.

Diagonalization of $\widehat{H}$ gave the energies of the exciton modes $\varepsilon_{e}$ as well as their associated eigenvectors $\psi_{e}$. Written in matrix notation, this looks like

$$
H=\sum_{e}\left|\psi_{e}\right\rangle \varepsilon_{e}\left\langle\psi_{e}\right|
$$

We obtained the excitonic transition dipole moment $\boldsymbol{\mu}_{e}$ which describe the strength and orientation of an excitonic modes in the system by

$$
\boldsymbol{\mu}_{e}=\sum_{m}^{N} \psi_{e, m} \boldsymbol{\mu}_{m}
$$

where $\psi_{e, m}$ are the expansion coefficients of the exciton states, $\left|\psi_{e}\right\rangle$, in the local mode basis. The linear absorbance spectra were calculated by summing stick spectra on a frequency grid according to the mode frequency, $\omega_{e}$, scaled by the transition dipole strength of the mode. Stick spectrum were then convolved with a Lorentzian lineshape to give the final spectra, according to

$$
I(\omega)=\sum_{e}\left|\boldsymbol{\mu}_{e}\right|^{2} \times \delta\left(\operatorname{round}\left(\omega-\omega_{e}\right)\right) \otimes \operatorname{IM}\left(\frac{-1}{\omega+i \gamma}\right)
$$

\section{B. Doorway state model}

Following the methods of Tokmakoff, we used Singular Value Decomposition (SVD) to decompose regions of the spectra with densely packed states into three orthogonal bright doorway modes which gives an overall representation of the origin of the signal. ${ }^{18}$ The set of excitonic transition dipoles within the spectral regions is first obtained by selecting a subset of $\psi_{e}$ whose associated energies fall within this region of interest

$$
\widetilde{\boldsymbol{\mu}}_{e}^{s u b}={ }_{s u b} \Psi_{e}^{\dagger} \widetilde{\boldsymbol{\mu}}_{m}
$$


Here we define $\widetilde{\boldsymbol{\mu}}_{m}$ as the $\mathrm{N} \times 3$ matrix whose rows are the local transition dipoles $\boldsymbol{\mu}_{m}$ and columns are the components of those vectors. Similarly, $\widetilde{\boldsymbol{\mu}}_{e}^{\text {sub }}$ is a $n \times 3$ matrix whose rows are the subset of exciton transition dipoles and columns are the components of the vectors. The matrix ${ }_{s u b} \Psi_{e}$ is an $\mathrm{N} \times n$ matrix which contains the subset of $\psi_{e}$ eigenvectors in its columns.

Next, matrix SVD is performed on $\widetilde{\boldsymbol{\mu}}_{e}^{s u b}$ to generate the decomposition:

$$
\widetilde{\boldsymbol{\mu}}_{e}^{\text {sub }}=U_{\text {sub }} S_{\text {sub }} V_{\text {sub }}^{T},
$$

where $S_{\text {sub }}$ is the diagonal matrix of singular values of the matrix $\widetilde{\boldsymbol{\mu}}_{e}^{\text {sub }}$ and $V_{\text {sub }}^{T}$ is the $3 \times 3$ matrix of the transition dipole moments for three bright doorway modes. $U_{\text {sub }}$ is the transformation matrix which builds $\widetilde{\boldsymbol{\mu}}_{e}^{\text {sub }}$ from the underlying doorway mode basis set. ${ }^{17}$ Thus we define $\widetilde{\boldsymbol{\mu}}_{d}^{\text {sub }}$ as the matrix of representative transition dipole moments of the spectral region referred to as the doorway transition dipoles,

$$
\widetilde{\boldsymbol{\mu}}_{d}^{\text {sub }} \equiv S_{\text {sub }} V_{\text {sub }}^{T}
$$

This can be rewritten using $U_{s u b}$ as the transformation matrix between $\widetilde{\boldsymbol{\mu}}_{e}^{\text {sub }}$ and $\widetilde{\boldsymbol{\mu}}_{d}^{\text {sub }}$ as

$$
\widetilde{\boldsymbol{\mu}}_{d}^{s u b}=U_{\text {sub }}^{T} \widetilde{\boldsymbol{\mu}}_{e}^{s u b} .
$$

We can then relate the contributions to the doorway transition dipoles back to local transition dipoles of the structure through the transformation matrix and the eigenvectors of the modes,

$$
\widetilde{\boldsymbol{\mu}}_{d}^{\text {sub }}=\Phi_{\text {sub }}^{T} \widetilde{\boldsymbol{\mu}}_{m},
$$

where $\Phi_{\text {sub }}={ }_{\text {sub }} \Psi_{e} U_{\text {sub }}$. The columns of $\Phi_{\text {sub }}$ give the amplitude and phase of the contribution of each local transition to the doorway mode. We applied the amplitudes and phases in $\Phi_{\text {sub }}$ back onto the local modes to plot the doorway modes in the main text. To obtain an average energy for the doorway mode, we used the transformation matrix $U_{s u b}$ which gives the contribution of each excitonic transition dipole to the doorway mode. We used the amplitude squared of the transformation

matrix, $U_{\text {sub }}{ }^{2}$, to weight the contribution of each exciton mode to the spectrum which we used to assign an average energy to each doorway mode,

$$
\varepsilon_{d}^{s u b}=\sum_{e} U_{s u b}^{2} \varepsilon_{e, d}^{s u b}
$$

\section{Nonlinear exciton scattering model}

The third-order response is obtained through the nonlinear exciton scattering model detailed by Mukamel and others..$^{20,50,51}$ Starting with Eq. (A5), we included an additional term which acts like a quartic expansion of an anharmonic potential,

$$
\widehat{H}=\sum_{m, n} \delta_{m n} \varepsilon_{m} b_{m}^{\dagger} b_{m}+\left(1-\delta_{m n}\right) \beta_{m n} b_{m}^{\dagger} b_{n}
$$




$$
+\sum_{m n, p q} \mathbb{U}_{m n, p q} b_{m}^{\dagger} b_{n}^{\dagger} b_{p} b_{q}-\sum_{m}\left(b_{m}^{\dagger}+b_{m}\right) \boldsymbol{\mu}_{m} \cdot \boldsymbol{E}(t)
$$

where $\mathbb{U}_{m n, p q}=\frac{\Delta}{4}\left(\delta_{m p} \delta_{n q}+\delta_{m q} \delta_{n p}\right)$ and $\Delta$ is the site anharmonic shift.

The third-order polarization can be calculated for the rephasing $\left(k_{R}=-k_{1}+k_{2}+k_{3}\right)$ and non-rephasing $\left(k_{N R}=+k_{1}-k_{2}+\right.$ $\left.k_{3}\right)$ signals,

$$
P_{v_{4}}^{k_{R}}\left(\tau_{3}, T_{2}, \tau_{1}\right)=\frac{1}{2^{3}} \exp \left(+i \phi_{3}+i \phi_{2}-i \phi_{1}\right) \sum_{v_{3} v_{2} v_{1}} S_{v_{4} v_{3} v_{2} v_{1}}^{k_{R}}\left(\tau_{3}, T_{2}, \tau_{1}\right) E_{v_{3}} E_{v_{2}} E_{v_{1}}
$$

and

$$
P_{v_{4}}^{k_{N R}}\left(\tau_{3}, T_{2}, \tau_{1}\right)=\frac{1}{2^{3}} \exp \left(+i \phi_{3}-i \phi_{2}+i \phi_{1}\right) \sum_{v_{3} v_{2} v_{1}} S_{v_{4} v_{3} v_{2} v_{1}}^{k_{N R}}\left(\tau_{3}, T_{2}, \tau_{1}\right) E_{v_{3}} E_{v_{2}} E_{v_{1}}
$$

Here, $P_{v_{4}}^{k_{R}}$ and $P_{v_{4}}^{k_{N R}}$ are the third order polarizations for the rephasing and non-rephasing diagrams, $\phi_{i}$ are the phases of the $i^{t h}$ pulses, $S_{v_{4} v_{3} v_{2} v_{1}}^{k_{R}}$ and $S_{v_{4} v_{3} v_{2} v_{1}}^{k_{N R}}$ are the non-linear response functions for the rephasing and non-rephasing diagrams, and $E_{v_{i}}$ are the electric fields, and $v_{i}$ are used to donate the polarization of the $i^{\text {th }}$ light interaction. The non-linear response functions depend on the wavevectors and the time intervals $\left(\tau_{3}=t_{4}-t_{3}, T_{2}=t_{3}-t_{2}, \tau_{1}=t_{2}-t_{1}\right)$ between pulses. The non-linear response function is obtained through propagating multiple excitations in the one-quantum exciton manifold. These responses are defined in terms of Green functions, which describe the forward propagation of under the influence of the Hamiltonian. The timedomain Green function is

$$
G(t)=\theta(t) \exp \left(-\frac{i}{\hbar} H(t)\right)
$$

where $\theta(t)$ is the Heaviside step function $(\theta(t)=0$ for $t<0, \theta(t)=1$ for $t \geq 0)$.

The Green function for each site can be calculated by transforming the excitonic Green function into the individual site basis,

$$
G_{m, n}(t)=\sum_{e} \psi_{e m} I_{e}(t) \psi_{e n}^{*}
$$

where the excitonic Green function gives the propagation of an excitation into an exciton mode,

$$
I_{e}(t)=\theta(t) \exp \left(-i \varepsilon_{e} t-\gamma_{e} t\right)
$$

where $\gamma_{e}$ is the dephasing rate for exciton $e$.

Tracking the propagation of each exciton excitation over each time interval where the excitation exists gives the non-linear response. The inclusion of the exciton scattering matrix $\Gamma_{e_{4} e_{3}, e_{2} e_{1}}$ allows for the interaction between excitations which allows for the non-linear signals to appear. The scattering is integrated over all possible mixing times giving a response of 


$$
\begin{gathered}
S_{v_{4} v_{3} v_{2} v_{1}}^{k}\left(\tau_{3}, T_{2}, \tau_{1}\right)=2 i \sum_{e_{4} e_{3} e_{2} e_{1}} \boldsymbol{M}_{e_{4} e_{3} e_{2} e_{2} \nu_{1} \nu_{3} v_{2} v_{1}} \times \int_{0}^{\tau_{3}+T_{2}} d \tau_{s}^{\prime \prime} \int_{0}^{\tau_{s}^{\prime \prime}} d \tau_{s}^{\prime} I_{e_{4}}\left(\tau_{s}^{\prime}\right) \times \\
I_{e_{3}}^{*}\left(\tau_{3}+T_{2}+\tau_{1}-\tau_{s}^{\prime}\right) \Gamma_{e_{4} e_{3}, e_{2} e_{1}}\left(\tau_{s}^{\prime \prime}-\tau_{s}^{\prime}\right) I_{e_{2}}\left(\tau_{3}+T_{2}-\tau_{s}^{\prime \prime}\right) I_{e_{1}}\left(\tau_{1}-\tau_{s}^{\prime \prime}\right) .
\end{gathered}
$$

Applying the time ordering of the pulses for the rephasing and non-rephasing signals gives,

$$
\begin{aligned}
S_{v_{4} v_{3} v_{2} v_{1}}^{k_{R}}\left(\tau_{3}, T_{2}, \tau_{1}\right)= & 2 i \sum_{e_{4} e_{3} e_{2} e_{1}} M_{e_{4} e_{3} e_{2} e_{1}}^{v_{4} v_{3} v_{2} v_{1}} \times \int_{0}^{\tau_{3}} d \tau_{s}^{\prime \prime} \int_{0}^{\tau_{s}^{\prime \prime}} d \tau_{s}^{\prime} I_{e_{4}}\left(\tau_{s}^{\prime}\right) I_{e_{1}}^{*}\left(\tau_{3}+T_{2}+\tau_{1}-\tau_{s}^{\prime}\right) \times \\
& \Gamma_{e_{4} e_{1}, e_{3} e_{2}}\left(\tau_{s}^{\prime \prime}-\tau_{s}^{\prime}\right) I_{e_{3}}\left(\tau_{3}-\tau_{s}^{\prime \prime}\right) I_{e_{2}}\left(\tau_{3}+T_{2}-\tau_{s}^{\prime \prime}\right)
\end{aligned}
$$

and

$$
\begin{gathered}
S_{v_{4} v_{3} v_{2} v_{1}}^{k_{N R}}\left(\tau_{3}, T_{2}, \tau_{1}\right)=2 i \sum_{e_{4} e_{3} e_{2} e_{1}} \boldsymbol{M}_{e_{4} e_{3} e_{2} e_{1}}^{v_{4} v_{3} v_{2} v_{1}} \times \int_{0}^{\tau_{3}} d \tau_{s}^{\prime \prime} \int_{0}^{\tau_{s}^{\prime \prime}} d \tau_{s}^{\prime} I_{e_{4}}\left(\tau_{s}^{\prime}\right) I_{e_{2}}^{*}\left(\tau_{3}+T_{2}-\tau_{s}^{\prime}\right) \times \\
\Gamma_{e_{4} e_{2}, e_{3} e_{1}}\left(\tau_{s}^{\prime \prime}-\tau_{s}^{\prime}\right) I_{e_{3}}\left(\tau_{3}-\tau_{s}^{\prime \prime}\right) I_{e_{1}}\left(\tau_{3}+T_{2}+\tau_{1}-\tau_{s}^{\prime \prime}\right) .
\end{gathered}
$$

$\boldsymbol{M}_{e_{4} e_{3} e_{2} e_{1}}^{v_{4} v_{3} v_{2} v_{1}}$ is defined as the orientationally averaged transition dipole strengths between the four local modes,

$$
\boldsymbol{M}_{e_{4} e_{3} e_{2} e_{1}}^{v_{4} v_{3} v_{2} v_{1}} \equiv\left\langle\left(\boldsymbol{\mu}_{e_{4}} \cdot \widehat{\boldsymbol{E}}_{v_{4}}\right)\left(\boldsymbol{\mu}_{e_{3}} \cdot \widehat{\boldsymbol{E}}_{v_{3}}\right)\left(\boldsymbol{\mu}_{e_{2}} \cdot \widehat{\boldsymbol{E}}_{v_{2}}\right)\left(\boldsymbol{\mu}_{e_{1}} \cdot \widehat{\boldsymbol{E}}_{v_{1}}\right)\right\rangle
$$

which we calculate using ${ }^{35}$

$$
\boldsymbol{M}_{e_{4} e_{3} e_{2} e_{1}}^{v_{4} v_{3} v_{2} v_{1}}=\frac{1}{30}\left|\mu_{e_{4}}\right|\left|\mu_{e_{3}}\right|\left|\mu_{e_{2}}\right|\left|\mu_{e_{1}}\right|\left(\begin{array}{l}
\cos \theta_{v_{1} v_{2}} \cos \theta_{v_{3} v_{4}} \\
\cos \theta_{v_{1} v_{3}} \cos \theta_{v_{2} v_{4}} \\
\cos \theta_{v_{1} v_{4}} \cos \theta_{v_{2} v_{3}}
\end{array}\right)^{T}\left(\begin{array}{ccc}
4 & -1 & -1 \\
-1 & 4 & -1 \\
-1 & -1 & 4
\end{array}\right)\left(\begin{array}{l}
\cos \theta_{e_{1} e_{2}} \cos \theta_{e_{3} e_{4}} \\
\cos \theta_{e_{1} e_{3}} \cos \theta_{e_{2} e_{4}} \\
\cos \theta_{e_{1} e_{4}} \cos \theta_{e_{2} e_{3}}
\end{array}\right)
$$

where $\cos \theta_{v_{i} v_{j}}$ describes the cosine of the angle between polarizations of light pulses $\widehat{\boldsymbol{E}}_{v_{i}}$ and $\widehat{\boldsymbol{E}}_{v_{j}}$, and $\cos \theta_{e_{i} e_{j}}$ describes the cosine of the angle between the transition dipole moments $\boldsymbol{\mu}_{e_{i}}$ and $\boldsymbol{\mu}_{e_{j}}$.

Fourier transforming $S^{k}$ along the $\tau_{1}$ and $\tau_{3}$, along with remembering contour integration gives the non-linear response functions in the mixed frequency-time domain which allows us to calculate the 2DIR spectrum, yields

$$
\begin{gathered}
S_{v_{4} v_{3} v_{2} v_{1}}^{k_{R}}\left(\omega_{3}, T_{2}, \omega_{1}\right)=2 i \sum_{e_{4} e_{3} e_{2} e_{1}} \boldsymbol{M}_{e_{4} e_{3} e_{2} \nu_{2} \nu_{1} \nu_{2} v_{2} v_{1}}^{v_{2}} \times I_{e_{1}}^{*}\left(T_{2}\right) I_{e_{2}}\left(T_{2}\right) \mathfrak{T}_{e_{4}}\left(\omega_{3}\right) \mathfrak{I}_{e_{1}}^{*}\left(-\omega_{1}\right) \times \\
\Gamma_{e_{4} e_{1}, e_{3} e_{2}}\left(\omega_{3}+\varepsilon_{e_{1}}+i \gamma_{e_{1}}\right) \mathcal{J}_{e_{3} e_{2}}\left(\omega_{3}+\varepsilon_{e_{1}}+i \gamma_{e_{1}}\right)
\end{gathered}
$$

and

$$
\begin{gathered}
S_{v_{4} \nu_{3} v_{2} v_{1}}^{k_{N R}}\left(\omega_{3}, T_{2}, \omega_{1}\right)=2 i \sum_{e_{4} e_{3} e_{2} e_{1}} \boldsymbol{M}_{e_{4} e_{3} e_{2} e_{1}}^{v_{4} v_{3} v_{2} v_{1}} \times I_{e_{1}}\left(T_{2}\right) I_{e_{2}}^{*}\left(T_{2}\right) \mathfrak{I}_{e_{4}}\left(\omega_{3}\right) \mathfrak{I}_{e_{1}}\left(\omega_{1}\right) \times \\
\Gamma_{e_{4} e_{2}, e_{3} e_{1}}\left(\omega_{3}+\varepsilon_{e_{2}}+i \gamma_{e_{2}}\right) \mathcal{J}_{e_{3} e_{1}}\left(\omega_{3}+\varepsilon_{e_{2}}+i \gamma_{e_{2}}\right) .
\end{gathered}
$$

The Fourier transformed Green functions are

$$
\mathfrak{I}_{e^{\prime}}(\omega)=\frac{i}{\omega-\varepsilon_{e^{\prime}}+i \gamma_{e^{\prime}}}
$$

and $\mathcal{J}_{e^{\prime} e^{\prime \prime}}$ is the two-exciton Green function 


$$
\mathcal{J}_{e^{\prime} e^{\prime \prime}}(\omega)=\frac{i}{\left.\omega-\varepsilon_{e^{\prime}-\varepsilon_{e} e^{\prime \prime}+i\left(\gamma_{e^{\prime}}+\gamma\right.} e^{\prime \prime}\right)}
$$

$\Gamma_{e_{4} e_{3}, e_{2} e_{1}}$ is the scattering of excitons written in an exciton basis. Calculating $\Gamma_{e_{4} e_{3}, e_{2} e_{1}}$, however, involves the interactions of excitations at local sites, thus we calculate $\Gamma_{e_{4} e_{3}, e_{2} e_{1}}$ by transforming from a site basis scattering matrix $\Gamma_{m, n}^{s i t e}$,

$$
\Gamma_{e_{4} e_{3}, e_{2} e_{1}}(\omega)=\sum_{m, n} \psi_{e_{4} m}^{*} \psi_{e_{3} m}^{*} \Gamma_{m, n}^{s i t e} \psi_{e_{2} n} \psi_{e_{1} n} .
$$

The size of $\Gamma_{e_{4} e_{3}, e_{2} e_{1}}$ is greatly reduced when we only consider elements where excitons $e_{4} e_{3}$ overlap and where excitons $e_{2} e_{1}$ overlap. We define an exciton overlap factor,

$$
\eta_{e, e^{\prime}}=\sum_{m}\left|\psi_{e, m}\right|\left|\psi_{e^{\prime}, m}\right|
$$

which relates the spatial overlap of each pair of excitons via the sites they occupy. We eliminate elements of $\Gamma_{e_{4} e_{3}, e_{2} e_{1}}$ by choosing a hard cutoff and only calculating $\Gamma_{e_{4} e_{3}, e_{2} e_{1}}$ where $\eta_{e_{4} e_{3}}$ and $\eta_{e_{2} e_{1}}$ are greater than the cutoff.

The site basis scattering matrix is calculated as

$$
\Gamma_{m, n}^{s i t e}(\omega)=-i \bar{\Delta}_{m}\left(\bar{D}^{-1}\right)_{m, n}
$$

where $\bar{\Delta}_{m}$ is the anharmonic shift at site $m$, and $\bar{D}$ is

$$
\bar{D}_{m, n}=\delta_{m n}+i \mathcal{G}_{m, n} \bar{\Delta}_{n}
$$

The matrix $\mathcal{G}_{m, n}$ is a two-exciton Green function written in the site basis,

$$
\mathcal{G}_{m, n}(\omega)=\sum_{e^{\prime}, e^{\prime \prime}} \psi_{e^{\prime} m} \psi_{e^{\prime \prime} m} \mathcal{J}_{e^{\prime} e^{\prime \prime}}(\omega) \psi_{e^{\prime} n}^{*} \psi_{e^{\prime \prime} n}^{*}
$$

In principle, $\mathcal{G}$ would be a tetradic matrix involving four sites. However, due to the local nature of the anharmonicity, meaning the site energy is only lowered on double excitations on the same site, we can reduce the size of $\mathcal{G}$ to an $\mathrm{N} \times \mathrm{N}$ matrix.

\section{ACKNOWLEDGMENTS}

ALS gratefully acknowledges support from the College of Science at the University of Notre Dame. DNE would like to thank the Notre Dame IBMS program and the Dana Wilson Quintero Memorial Graduate Fellowship for their support in this project. We would like to thank Allen Oliver for discussions regarding

\section{REFERENCES}

${ }^{1}$ T. MIYAZAWA, J. Chem. Phys. 32, 1647 (1960).

${ }^{2}$ S. Krimm and J. BANDEKAR, Adv. Protein Chem. 38, 181 (1986). 
${ }^{3}$ H. Torii and M. Tasumi, J. Raman Spectrosc. 29, 81 (1998).

${ }^{4}$ A. Barth and C. Zscherp, Q. Rev. Biophys. 35, 369 (2002).

${ }^{5}$ J.W. Brauner, C. Dugan, and R. Mendelsohn, J. Am. Chem. Soc. 122, 677 (2000).

${ }^{6}$ J. Kubelka and T.A. Keiderling, J. Am. Chem. Soc. 123, 12048 (2001).

${ }^{7}$ G. Zandomeneghi, M.R.H. Krebs, M.G. McCammon, and M. Fändrich, Protein Sci. 13, 3314 (2009).

${ }^{8}$ S.A. Oladepo, K. Xiong, Z. Hong, S.A. Asher, J. Handen, and I.K. Lednev, Chem. Rev. 112, 2604 (2012).

${ }^{9}$ K.M. Towle, C.T. Lohans, M. Miskolzie, J.Z. Acedo, M.J. van Belkum, and J.C. Vederas, Biochemistry 55, 4798 (2016).

${ }^{10}$ E. Tayeb-Fligelman, O. Tabachnikov, A. Moshe, O. Goldshmidt-Tran, M.R. Sawaya, N. Coquelle, J.-P. Colletier, and M. Landau, Science (80-. ). 355, 831 (2017).

${ }^{11}$ E. Tayeb-Fligelman, N. Salinas, O. Tabachnikov, and M. Landau, Structure 28, 301 (2020).

${ }^{12}$ S.A. Cracchiolo O, Edun D, Betti V, Goldberg J, ChemRxiv. Cambridge Cambridge Open Engag. (2021).

${ }^{13}$ H. Torii and M. Tasumi, J. Chem. Phys. 96, 3379 (1992).

${ }^{14}$ S.H. Lee and S. Krimm, Chem. Phys. 230, 277 (1998).

15 Z. Ganim and A. Tokmakoff, Biophys. J. 91, 2636 (2006).

${ }^{16}$ H. Torii and M. Tasumi, J. Chem. Phys. 97, 92 (1992).

${ }^{17}$ H. Torii and M. Tasumi, J. Chem. Phys. 97, 86 (1992).

${ }^{18}$ H.S. Chung and A. Tokmakoff, J. Phys. Chem. B 110, 2888 (2006).

${ }^{19}$ D. Abramavicius and S. Mukamel, J. Chem. Phys. 122, 134305 (2005).

${ }^{20}$ D. Abramavicius and S. Mukamel, Chem. Phys. 318, 50 (2005).

${ }^{21}$ S. Mukamel, Principles of Nonlinear Optical Spectroscopy (Oxford University Press, New York, New York, 1998).

${ }^{22}$ W. Zhuang, D. Abramavicius, T. Hayashi, and S. Mukamel, J. Phys. Chem. B 110, 3362 (2006).

${ }^{23}$ J.W.S. Hearle, J. Appl. Polym. Sci. 7, 1175 (1963).

${ }^{24}$ J. Wang and R.M. Hochstrasser, Chem. Phys. 297, 195 (2004).

${ }^{25}$ S. Mukherjee, P. Chowdhury, and F. Gai, J. Phys. Chem. B 111, 4596 (2007).

${ }^{26}$ A.M. Dwivedi and S. Krimm, J. Phys. Chem. 88, 620 (1984).

${ }^{27}$ J.H. Choi, S. Ham, and M. Cho, J. Chem. Phys. 117, 6821 (2002).

${ }^{28}$ S. Woutersen and P. Hamm, J. Chem. Phys. 115, 7737 (2001).

${ }^{29}$ M.R. Panman, C.N. van Dijk, H. Meuzelaar, and S. Woutersen, J. Chem. Phys. 142, 041103 (2015).

${ }^{30}$ L. Minnes, D.J. Shaw, B.P. Cossins, P.M. Donaldson, G.M. Greetham, M. Towrie, A.W. Parker, M.J. Baker, A.J. Henry, R.J. Taylor, and N.T. Hunt, Anal. Chem. 89, 10898 (2017). 
${ }^{31}$ M. Khalil, N. Demirdöven, and A. Tokmakoff, J. Phys. Chem. A 107, 5258 (2003).

32 S. Woutersen and P. Hamm, J. Phys. Chem. B 104, 11316 (2000).

${ }^{33}$ D.Y. Vorobyev, C.-H. Kuo, D.G. Kuroda, J.N. Scott, J.M. Vanderkooi, and R.M. Hochstrasser, J. Phys. Chem. B 114, 2944 (2010).

${ }^{34}$ R.M. Hochstrasser, Chem. Phys. 266, 273 (2001).

${ }^{35}$ P. Hamm and M. Zanni, Concepts and Methods of 2D Infrared Spectroscopy (Cambridge University Press, Cambridge, 2011).

${ }^{36}$ V. Cervetto, J. Helbing, J. Bredenbeck, and P. Hamm, J. Chem. Phys. 121, 5935 (2004).

${ }^{37}$ A. Huerta-Viga and S. Woutersen, J. Phys. Chem. Lett. 4, 3397 (2013).

${ }^{38}$ M.R. Panman, C.N. van Dijk, H. Meuzelaar, and S. Woutersen, J. Chem. Phys. 142, 041103 (2015).

${ }^{39}$ O.M. Cracchiolo, D.N. Edun, V.M. Bretti, J.M. Goldberg, and A.L. Serrano, Submitt. Publ. (n.d.).

${ }^{40}$ S. Irgen-Gioro, K. Gururangan, R.G. Saer, R.E. Blankenship, and E. Harel, Chem. Sci. 10, 10503 (2019).

${ }^{41}$ M.J. Nee, C.R. Baiz, J.M. Anna, R. McCanne, and K.J. Kubarych, J. Chem. Phys. 129, 084503 (2008).

${ }^{42}$ M. Khalil, N. Demirdöven, A. Tokmakoff, N. Demirdöven, and A. Tokmakoff, J. Chem. Phys. 121, 362 (2004).

${ }^{43}$ Y.-C. Cheng and G.R. Fleming, J. Phys. Chem. A 112, 4254 (2008).

44 T.R. Calhoun, N.S. Ginsberg, G.S. Schlau-Cohen, Y.-C. Cheng, M. Ballottari, R. Bassi, and G.R. Fleming, J. Phys. Chem. B 113, 16291 (2009).

${ }^{45}$ E.A. Arsenault, Y. Yoneda, M. Iwai, K.K. Niyogi, and G.R. Fleming, Nat. Commun. 11, 1460 (2020).

${ }^{46}$ E. Thyrhaug, K. Žídek, J. Dostál, D. Bína, and D. Zigmantas, J. Phys. Chem. Lett. 7, 1653 (2016).

${ }^{47}$ E. Thyrhaug, R. Tempelaar, M.J.P. Alcocer, K. Žídek, D. Bína, J. Knoester, T.L.C. Jansen, and D. Zigmantas, Nat. Chem. 10, 780 (2018).

${ }^{48}$ J. Cao, R.J. Cogdell, D.F. Coker, H.-G. Duan, J. Hauer, U. Kleinekathöfer, T.L.C. Jansen, T. Mančal, R.J.D. Miller, J.P. Ogilvie, V.I. Prokhorenko, T. Renger, H.-S. Tan, R. Tempelaar, M. Thorwart, E. Thyrhaug, S. Westenhoff, and D. Zigmantas, Sci. Adv. 6, eaaz4888 (2020).

${ }^{49}$ J. Lim, D. Paleček, F. Caycedo-Soler, C.N. Lincoln, J. Prior, H. von Berlepsch, S.F. Huelga, M.B. Plenio, D. Zigmantas, and J. Hauer, Nat. Commun. 6, 7755 (2015).

${ }^{50}$ D. Abramavicius, B. Palmieri, D. V. Voronine, F. Šanda, and S. Mukamel, Chem. Rev. 109, 2350 (2009).

${ }^{51}$ C.M. Cheatum, A. Tokmakoff, and J. Knoester, J. Chem. Phys. 120, 8201 (2004). 